\title{
BASIC SCIENCE ARTICLE Lyophilization of human amniotic fluid is feasible without affecting biological activity
}

\author{
John I. Coon ${ }^{1}$, Sangeeta Jain ${ }^{2}$, Krishna M. Sepuru ${ }^{3}$, Yerin Chung ${ }^{4}$, Krishnan Mohankumar ${ }^{4}$, Krishna Rajarathnam ${ }^{3}$ and Sunil K. Jain ${ }^{1}$
}

BACKGROUND: Fetal swallowing of human amniotic fluid (hAF) containing trophic factors (TFs) promotes gastrointestinal tract (GIT) development. Preterm birth interrupts hAF swallowing, which may increase the risk of necrotizing enterocolitis (NEC). Postnatally, it is difficult to replicate fetal swallowing of hAF due to volume. We aimed to evaluate whether hAF lyophilization is feasible and its effect on hAF-borne TFs.

METHODS: We collected hAF ( $n=16)$ from uncomplicated pregnancies. hAF was divided into three groups: unprocessed control (C), concentration by microfiltration (F), and by dialysis and lyophilization (L). EGF, HGF, GM-CSF, and TGF-a were measured in each group by multiplex assay. Bioavailability of TFs was measured by proliferation and LPS-induced IL- 8 production by intestinal epithelial cells FHs74.

RESULTS: After dialysis/lyophilization, GM-CSF and TGF-a were preserved with partial loss of EGF and HGF. hAF increased cell proliferation and reduced LPS-induced IL-8 production compared to medium alone. Compared to control, dialysis/lyophilization and filtration of hAF increased FHs74 cell proliferation $(p<0.001)$ and decreased LPS-induced IL-8 production $(p<0.01)$. CONCLUSIONS: Lyophilization and filtration of hAF is feasible with partial loss of TFs but maintains and even improves bioavailability of TFs measured by proliferation and LPS-induced IL-8 production by FHs74.

Pediatric Research (2020) 87:847-852; https://doi.org/10.1038/s41390-019-0632-0

\section{INTRODUCTION}

Development and maturation of gastrointestinal tract (GIT) is a complex process. Fetal intestinal epithelial cell (IEC) morphogenesis, differentiation, and maturation depend on the activity of various trophic factors (TFs), peptides, and cytokines present in the swallowed amniotic fluid (AF). ${ }^{1}$ Fetal human AF (hAF) swallowing is detected at 16 week's gestation, which exposes GIT to many bioactive molecules in hAF. Hirai et al. $^{2}$ demonstrated that the trophic effects of hAF were equivalent to human milk. They further showed that hAF contains significant levels of epithelial growth factor (EGF), insulin-like growth factor 1 (IGF-1), transforming growth factor-alpha (TGF-a), hepatocyte growth factor (HGF), fibroblast growth factor (FGF), and vasoactive endothelial growth factor. hAFborne TFs work together in concert to exert their bioactivity. The concentrations of these TFs change with the gestation to have important and very specific functions in the development and maturation of GIT. At mid-gestation (around 24-26 weeks), secretory and absorptive functions are only partially developed. ${ }^{3}$

Human EGF, molecular weight (MW) of $\sim 6 \mathrm{kDa}$, is a single-chain polypeptide consisting of 48 amino acids with 3 disulfide bonds. ${ }^{4}$ Human TGF-a is structurally similar to EGF and is also a singlechain polypeptide consisting of 50 amino acids with 3 disulfide bonds with $\mathrm{MW}$ of $\sim 6 \mathrm{kDa}$. ${ }^{5} \mathrm{HGF}$ is a heterodimer composed of a $\sim 69 \mathrm{kDa}$ a-subunit and a $\sim 34 \mathrm{kDa} \beta$-subunit. ${ }^{6}$ Granulocyte macrophage colony-stimulating factor (GM-CSF) is a $\sim 14 \mathrm{kDa}$ globular protein consisting of 127 amino acids with 2 disulfide bonds. ${ }^{7}$ Size of TFs determined the type of filter and dialysis membrane necessary to retain TFs during concentration of hAF. See Table 1 for summary of the studied TFs and their sizes and roles in intestinal development.

Necrotizing enterocolitis (NEC) is the leading cause of morbidity and mortality from GIT disease in premature infants. ${ }^{8}$ The cause of NEC remains unclear, but current evidence suggests multifactorial likely mucosal barrier interruption leading to bacterial translocation of gut, which triggers inflammatory cascade. ${ }^{8}$ Leaphart et al. ${ }^{9}$ have shown that the lipopolysaccharide (LPS) receptor, Toll-like receptor 4 (TLR4), has an important role in the pathogenesis of NEC by increased TLR4 activation. In an animal model of NEC, Sodhi et al. ${ }^{10}$ showed that TLR4 null mice had decreased intestinal mucosal injury and enhanced healing. ${ }^{9}$ These findings provide strong evidence for a pivotal role for TLR4 in NEC. In an animal model of NEC, Good et al. ${ }^{11}$ showed AF-borne EGF has inhibitory effects on TLR4 signaling leading to reduced severity of NEC via EGFR activation. We also showed decreased incidence and severity of experimental NEC in rat pups by enteral supplementation of $A F$ mediated, at least in part, by HGF. ${ }^{12}$

The above studies led us to the hypothesis that premature infants are more prone to develop NEC and hAF supplementation may offer a unique and an innovative measure to prevent NEC in preterm infants. In utero, term human fetus swallows 500-1000 $\mathrm{ml} /$ day of $\mathrm{hAF}^{13,14}$ and about $161-285 \mathrm{ml} /$ day at $25-30$ weeks gestation. ${ }^{14}$ After preterm birth, there is abrupt interruption of fetal hAF swallowing, which results in reduced TF levels in the developing premature intestine. We speculate preterm delivery due to lack of protective TFs predispose preterm infants to develop NEC, a devastating disease.

\footnotetext{
${ }^{1}$ Department of Pediatrics, Division of Neonatology, University of Texas Medical Branch, Galveston, TX, USA; ${ }^{2}$ Department of Obstetrics and Gynecology, Division of Maternal Fetal

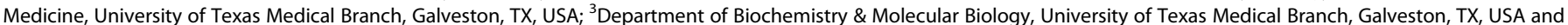
${ }^{4}$ Department of Pediatrics, Division of Neonatology, John Hopkins University, Baltimore, MD, USA

Correspondence: Sunil K. Jain (skjian@utmb.edu)
}

Received: 5 June 2019 Revised: 20 September 2019 Accepted: 2 October 2019

Published online: 22 November 2019 
Table 1. Studied human amniotic fluid-borne trophic factor sizes and roles in the gastrointestinal tract.

\begin{tabular}{lll}
\hline Trophic factor & Molecular weight & Role in intestinal development \\
\hline EGF & $\sim 6 \mathrm{kDa}$ & Stimulates intestinal epithelial cell mitosis and proliferation \\
GM-CSF & $\sim 14 \mathrm{kDa}$ & Involved in epithelial cell maintenance \\
HGF & $\sim 69 \mathrm{kDa} \alpha$-subunit and $\sim 34 \mathrm{kDa} \beta$-subunit & Involved in intestinal cell proliferation \\
TGF- $\alpha$ & $\sim 6 \mathrm{kDa}$ & Primary role may be involved in intestinal mucosal repair \\
\hline EGF epidermal growth factor, $G M-C S F$ granulocyte macrophage colony-stimulating factor, $H G F$ hepatocyte growth factor, TGF transforming growth factor
\end{tabular}

As we $\mathrm{e}^{12}$ and others ${ }^{2}$ have shown that different TFs (EGF, HGF, etc.) are protective against NEC in preterm rodent model of NEC, we propose that it could be possible to prevent NEC in preterm infants by enteral supplementation of mother's own AF during early neonatal period. To simulate fetal hAF swallowing, we have to supplement about 150-200 ml/day of hAF, which will be $\sim 5-10$ $\mathrm{ml} / \mathrm{h}$. However, it is unrealistic to supplement such high volumes of enteral hAF in preterm infants. To reduce the volume of enteral supplementation of hAF, we explored different protocols including dialysis followed by lyophilization that allows reconstitution of hAF in a practically acceptable volume. We sought to find out whether lyophilization of hAF is feasible without losing bioavailability of TFs in hAF.

\section{METHODS}

\section{Subjects}

After approval of institutional review board (IRB 06-323) of University of Texas Medical Branch, Galveston, TX, hAF was collected from the women with normal term pregnancy who had repeat cesarean section. Consent was not needed because hAF is considered to be a medical waste and discarded as de-identified waste. Subjects with any complication of pregnancy including prematurity, multiple gestations, fetal abnormalities on prenatal ultrasound, chromosomal anomalies of prenatal testing, maternal diabetes, pregnancy-induced hypertension, pre-eclampsia, abruption, urinary tract infections, history of drug use during pregnancy, chorioamnionitis, meconium-stained $\mathrm{AF}$, or breech presentation were excluded from the study.

hAF collection

AF was collected by a trained obstetrician (Sangeeta Jain) before baby was delivered by cesarean section. After uterine incision, a small incision was made to the amniotic membrane through which a sterile catheter was introduced in the uterine cavity. By using a sterile syringe, $20-25 \mathrm{ml}$ of sterile $\mathrm{AF}(n=16)$ was collected in a sterile container. To remove cells and debris, AF was centrifuged at $12,000 \mathrm{RPM}$ for $10 \mathrm{~min}$ at $4^{\circ} \mathrm{C}$. The supernatant was divided into 3 aliquots ( $5 \mathrm{ml}$ each) and immediately stored at $-80^{\circ} \mathrm{C}$ for future use. All AF samples were stored in aliquots and conditions were monitored according to the Biospecimen Reporting for Improved Study Quality guidelines. ${ }^{15}$ The first aliquot was used as a control, the second sample was dialyzed and lyophilized and reconstituted, while the third sample was concentrated by ultrafiltration with a micro-filter and reconstituted.

Experimental groups

Each hAF sample was divided into 3 aliquots of $5 \mathrm{ml}$ each and stored at $-80^{\circ} \mathrm{C}$. One aliquot of hAF was kept unprocessed (control-C group), the second aliquot was filtered (filter group-F group) and the last hAF sample was lyophilized (lyophilized-L group). All samples were saved at $-80^{\circ} \mathrm{C}$ for future studies.

Quantification and bioavailability of TFs in hAF

For the quantification of TFs in hAF, we measured the presence of different TFs by bead assay using BioPlex. hAF is known to increase IEC proliferation and to decrease LPS-induced interleukin (IL)-8 production by IEC. For the bioavailability of TFs in hAF, we measured the IEC proliferation and LPS-induced IL-8 production in different groups and compared it with the culture medium.

\section{Filtration of hAF}

Filtration was used as a means for concentrating hAF-borne TFs. A $5-\mathrm{ml}$ sample of hAF was placed in a centrifugal filter device, namely, Amicon Ultra-15 (ThermoFisher Scientific, Waltham, MA), with a 3-kiloDalton (kDa) MW cut-off (MWCO) and centrifuged at $4500 \mathrm{RPM}$ for $150 \mathrm{~min}$ at $4{ }^{\circ} \mathrm{C}$ yielding a final volume of $\sim 0.5 \mathrm{ml}$. Phosphate-buffered saline (PBS) was added to bring it back to a prefiltration volume of $5 \mathrm{ml}$. Samples were stored at $-80^{\circ} \mathrm{C}$ for further use.

\section{Lyophilization of hAF}

Samples were first dialyzed to reduce the concentrations of small MW components including various cations and anions. hAF was thawed at room temperature and $5 \mathrm{ml} \mathrm{hAF}$ was placed in Slide-ALyzer $^{\mathrm{TM}}$ (ThermoFisher Scientific, Waltham, MA) dialysis cassettes with a $2 \mathrm{kDa}$ MWCO with sterile syringe. Samples were then serially dialyzed against $10 \%$ PBS buffer for $8 \mathrm{~h}$ with two exchanges. Samples were then placed in $15-\mathrm{ml}$ centrifuge tubes (Globe Scientific Inc., Mahwah, NJ) and flash frozen in a mixture of $\sim$ 1:1 acetone and dry ice and lyophilized using Freezone 4.5 plus (Labconco, Kansas City, MO) for $24 \mathrm{~h}$. The lyophilized samples were then reconstituted in $5 \mathrm{ml}$ of PBS buffer and stored at $-80^{\circ} \mathrm{C}$ for further use.

\section{Measurement of TFs in hAF}

TF levels were measured (triplicate) of all three groups by a magnetic bead-based multiplex assay (BioPlex array, Bio-Rad, Hercules, CA) that included EGF, HGF, and TGF-a. These factors were selected based on existing knowledge and their effects on intestinal epithelium. ${ }^{16,17}$

\section{IEC proliferation}

We investigated IEC proliferation activity of all samples using primary small IECs, FHs74 (ATCC, Manassas, VA). The epithelial cells were plated in 96 -well plates at a density of $1-2 \times 10^{4}$ per well. Growth media consisted of Dulbecco's modified Eagle's medium (DMEM) containing $4 \mathrm{mM}$ L-glutamine, $1.5 \mathrm{~g} / \mathrm{l}$ sodium bicarbonate, $4.5 \mathrm{~g} / \mathrm{l}$ glucose, $50 \mathrm{U} / \mathrm{ml}$ penicillin, and $50 \mu \mathrm{g} / \mathrm{ml}$ streptomycin incubated at $37^{\circ} \mathrm{C}$ with $5 \% \mathrm{CO}_{2}$. Media were changed $24 \mathrm{~h}$ after plating and then every $72 \mathrm{~h}$. After $24 \mathrm{~h}$, culture media were changed to phenol red-free media containing $30 \%$ (volume/ volume) hAF (control, lyophilized, or filtered). After $48 \mathrm{~h}$, cells were labeled overnight with bromodeoxyuridine (BrdU) and cell proliferation was measured by use of a commercially available BrdU ELISA Kit (Roche, Indianapolis, IN). Briefly, cells were fixed and treated with a peroxidase-conjugated mouse monoclonal anti-BrdU antibody. BrdU incorporation was quantified by adding tetramethyl benzidine solution (substrate) and measuring absorbance at $490 \mathrm{~nm}$. In this assay, absorbance is related logarithmically to cell number. Each sample was performed in quadruplicates. 
Suppression of inflammatory response with hAF

IL-8 was measured selectively because of its reported role in modulating LPS-mediated inflammatory processes and the fact that this chemokines is significantly induced by LPS in NEC models. ${ }^{11}$ Human small intestine epithelial cells FHs74 (ATCC, Manassas, VA) were cultured to $80 \%$ confluence in 6 -well plates. Standard growth media (DMEM) and conditions described by the manufacturer were used. The media was changed initially at $24 \mathrm{~h}$ and then every $72 \mathrm{~h}$. Once $80 \%$ confluence was achieved, the cells were incubated with media containing $50 \mathrm{ng} / \mathrm{ml}$ LPS (SigmaAldrich, St. Louis, MO) in the presence or absence of $30 \%$ (vol/vol) hAF (control, dialyzed/lyophilized, or filtered) for $4 \mathrm{~h}$, based on a previously performed LPS time course. Inflammatory cytokine mRNA expression was measured by quantitative reverse transcription polymerase chain reaction (qRT-PCR) as described previously. ${ }^{18}$ Briefly, total cellular RNA was extracted using Tri Reagent (Sigma) and $2 \mu \mathrm{g}$ of RNA was used for RT by Super Script III First-Strand Synthesis System from Invitrogen (Carlsbad, CA). One microliter of cDNA products were amplified in a 10- $\mu$ l reaction system containing $5 \mu \mathrm{l}$ of iQ SYBR Green Supermix (Bio-Rad) and $400 \mathrm{nM}$ primer mixtures. All reactions were processed in a MyiQ Single Color Real-Time PCR thermocycler using a two-step plus melting curve program. Results were analyzed by the iQ5 program (Bio-Rad) and the data were analyzed using the $\triangle C T$ method in reference to glyceraldehyde 3-phosphate dehydrogenase. Validated primers were purchased from Qiagen (Germantown, MD). Each sample was performed in quadruplicates.

Statistical analysis

Data were expressed as mean \pm standard deviation (SD) and analyzed by the SPSS software version 25 (SPSS Inc., Chicago, IL). For comparison between two variables, the paired $T$ test was used. For comparison of more than two variables, one-way analysis of variance followed by post hoc least significance difference test was utilized. For all tests, $p<0.05$ was considered statistically significant.

\section{RESULTS}

Collection and lyophilization

We obtained 16 samples of hAF and divided them into three groups: unprocessed hAF control (C), filtered hAF (F), and lyophilized hAF (L).

TF quantification

Levels of EGF, HGF, GM-CSF, and TGF-a quantified by BioPlex bead assay are shown in Fig. 1. The mean \pm SD of EGF for the 16 samples was $670 \pm 403,422 \pm 358$, and $373 \pm 165 \mathrm{pg} / \mathrm{ml}$ for the control, filtered, and dialyzed/lyophilized groups, respectively $(p=0.03)$. Level of EGF was $57 \%$ in lyophilization vs control $(p=0.006)$ but no significant difference in filtered vs lyophilized hAF samples $(p=0.51)$. The average HGF was $4719 \pm 1754,2315 \pm 1733$, and $2572 \pm 1487 \mathrm{pg} / \mathrm{ml}$ for the control, filtered, and lyophilized groups, respectively $(p<0.001)$. The level of HGF was $55 \%$ in lyophilization from control $(p<0.001)$ and no significance difference between filtered vs lyophilized hAF samples $(p=0.40)$. The average GM-CSF was $87 \pm 51,80 \pm 57$, and $83 \pm 58 \mathrm{pg} / \mathrm{ml}$ for the control, filtered, and lyophilized groups, respectively $(p=0.93)$, with $95 \%$ recovery of GM-CSF in lyophilization compared to control $(p=0.54)$. The average TGF-a was $9.2 \pm 4.9,7.8 \pm 4.6$, and $7.2 \pm 4.7 \mathrm{pg} / \mathrm{ml}$ for the control, filtered, and lyophilized groups, respectively $(p=0.93)$, with $78 \%$ preservation of TGF-a in lyophilization compared to control $(p=0.12)$.

IEC proliferation

We and others ${ }^{2,12}$ have shown increased IEC proliferation by hAFborne TFs. We then sought to determine the bioavailability of hAF-
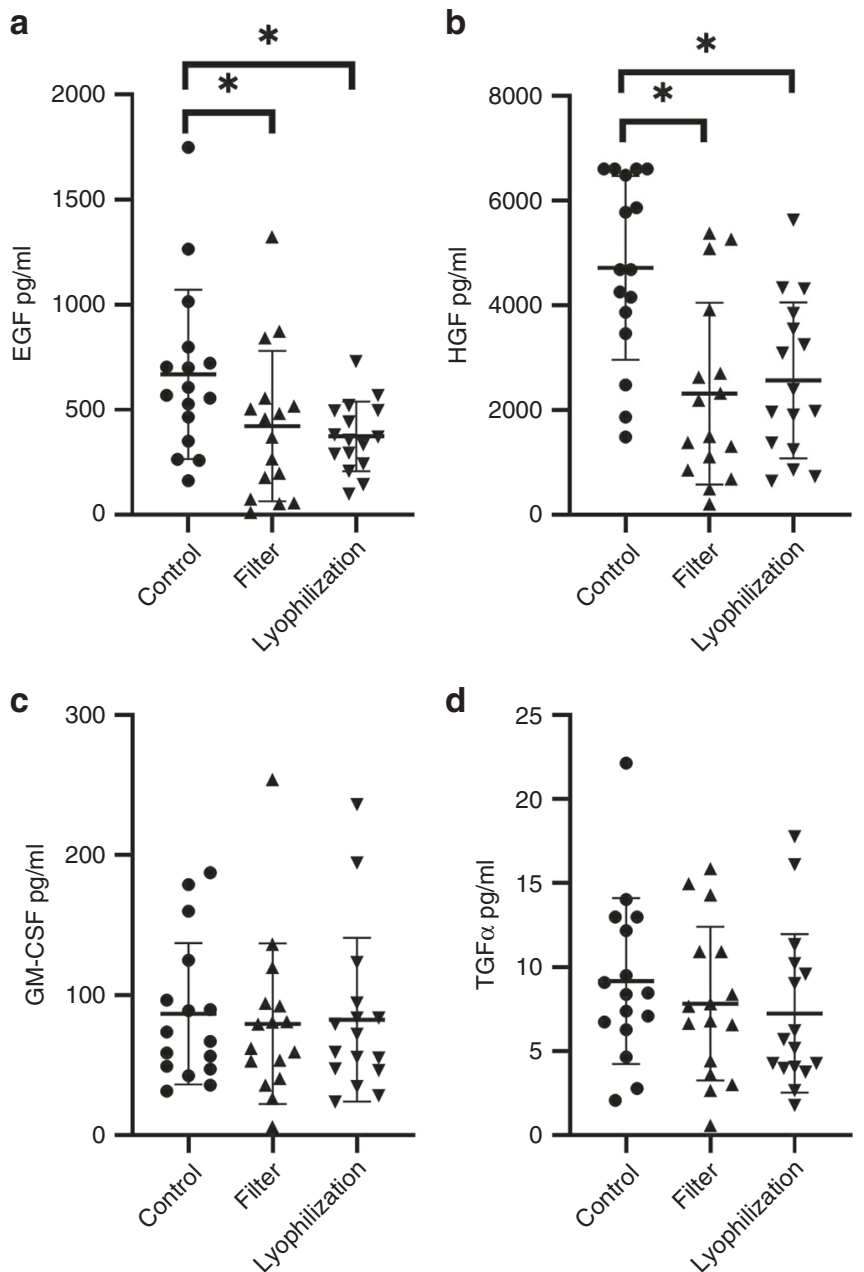

Fig. 1 Quantification of trophic factors (TFs) via BioPlex bead assay from three preparations of human amniotic fluid (hAF): unprocessed control (C), filtration (F), and lyophilization (L) a Epidermal growth factor (EGF); $\left({ }^{*} p<0.05\right.$, significant difference in $C$ vs $F$ and L. No significance (NS) difference in F vs L). b Hepatocyte growth factor (HGF); $\left({ }^{*} p<0.05\right.$, significant difference in C vs F and L. NS difference in $\mathrm{F}$ vs L). $c$ Granulocyte macrophage colony stimulating factor (GMCSF); NS in GM-CSF among all preparations. d Transforming growth factor-alpha (TGF- $\alpha$ ); NS difference in TGF- $\alpha$ among all preparations. All TFs were measured in triplicate and the data represent means of the triplicate.

borne TFs of lyophilized and filtered hAF compared to control hAF. We chose to use a human small IEC line, FHs74, to measure the bioactivity of the three preparation of hAF. Pooled samples of control, filtered, and lyophilized hAF preparations were cultured with BrdU, a thymidine analog, for incorporation into cellular DNA during replication. After fixation and treatment with peroxidaseconjugated mouse monoclonal anti-BrdU antibodies, exposure to tetramethyl benzidine solution allowed for quantification of cells via measuring absorbance at $490 \mathrm{~nm}$. Differences in absorbance for the three preparations performed in quadruplicates are shown in Fig. 2. Mean $\pm S D$ of absorbance at $490 \mathrm{~nm}$ was measured as $0.221 \pm 0.020,0.500 \pm 0.15$, and $0.560 \pm 0.18$, respectively $(p<$ $0.001)$, showing an increase in cellular proliferation of filtered $(p<0.001)$ and dialyzed/lyophilized samples compared to the control $(p<0.001)$. Despite some loss in TF levels after lyophilization, we demonstrated preserved and even increased cellular proliferation due to lyophilization of hAF. 


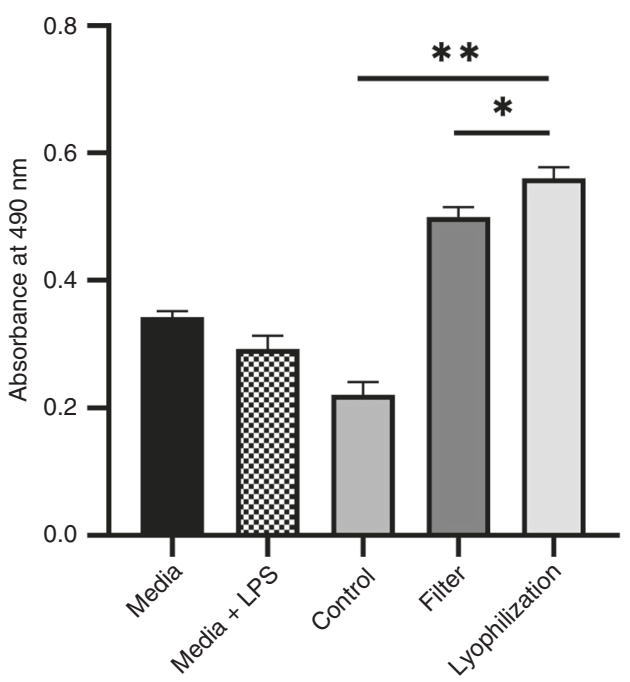

Fig. 2 Cellular proliferation of FHs74, small intestinal epithelial cell line, as measured by absorbance at $490 \mathrm{~nm}$ of growth media, lipopolysaccharide, and three preparations of human amniotic fluid (hAF): control (C), filtered (F), and lyophilized (L). $\quad\left({ }^{*} p<0.01\right.$ represents significant difference in IEC proliferation in $C$ vs $F$ vs $L$; ${ }^{* *} p<0.001$ represents significant difference in IEC proliferation in $\mathrm{L}$ vs F). The data represent mean of quadruplicate measurements.

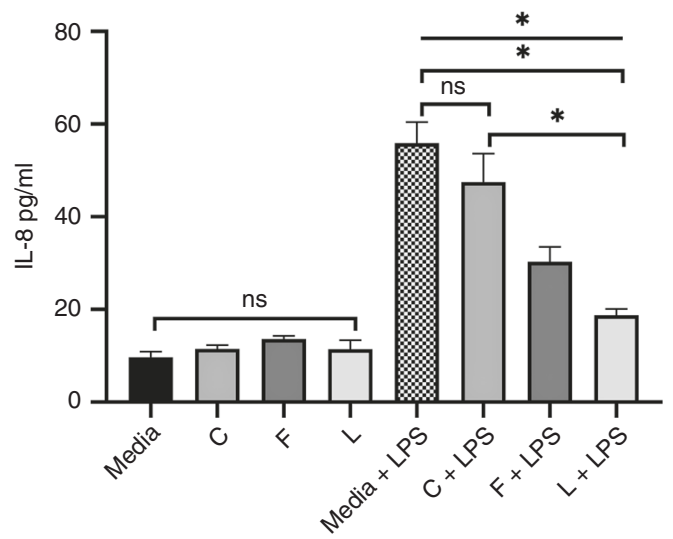

Fig. 3 Comparison of interleukin 8 (IL-8) expression in growth media, growth media with lipopolysaccharide (LPS), three preparations of human amniotic fluid (hAF): control (C), filtered (F), lyophilized (L), and three preparations of hAF with LPS: C+LPS, F + LPS, and L+LPS. No difference between IL-8 levels in lyophilization vs media alone $(p=0.19)$. A difference in IL-8 reduction exists among the group of media and preparations of hAF with LPS represented by a line $\left({ }^{*} p<0.001\right)$. There are significant reductions in L+LPS when compared to media with LPS and C+LPS $\left({ }^{*} p<0.001\right.$ for both), represented by brackets. There was no significant reduction in C+LPS when compared to media with LPS, represented by bracket $(p=0.19)$.

\section{Suppression of inflammatory response with hAF}

We sought to further characterize the bioactivity of dialyzed/ lyophilized hAF-borne TFs via a NEC-like model with LPS-induced activation of TLR4. We tested FHs74 IEC cultures in the aforementioned growth media alone and with the three preparations of hAF, each with and without addition of LPS, and determined IL-8 concentration via qRT-PCR (Fig. 3). Each sample was measured in quadruplicate. IL-8 is expressed in small amounts even in the absence of LPS. In the samples without LPS introduction, mean IL-8 levels were measured at 9.7 $\pm 1.2,11.5 \pm$ $4.5,13.7 \pm 0.8$, and $11.5 \pm 1.9 \mathrm{pg} / \mathrm{ml}$ for media, control, filtered, and lyophilized samples, respectively, with variation detected $(p<$
0.01). There was, however, no difference in IL-8 concentration in dialyzed/lyophilized hAF compared with media alone $(p=0.19)$ or when compared to unprocessed hAF ( $p=0.97)$. This suggests that no additional endotoxin exposure occurred throughout the handling and lyophilizing of hAF. Samples exposed to LPS showed a mean IL-8 production of $55.9 \pm 4.5,47.5 \pm 6.1,30.3 \pm 3.2$, and $18.8 \pm 1.3 \mathrm{pg} / \mathrm{ml}$ for media with LPS, control with LPS (C+LPS), filtered with LPS $(F+L P S)$, and lyophilized with LPS (L+LPS) samples, respectively, with variation detected $(p<0.001)$. When compared to the growth media plus LPS, C+LPS showed reduction, but it was not significant $(p=0.19)$, but there was significant reduction with $\mathrm{F}+\mathrm{LPS}$ and $\mathrm{L}+\mathrm{LPS}(p<0.01$ and $p<$ 0.001 , respectively). L+LPS compared to $C+$ LPS also showed significant reduction $(p<0.001)$. Again, despite some loss in TF levels, we demonstrate significant reduction of IL-8 production. Additional studies are necessary to understand the molecular basis underlying enhanced anti-inflammatory effects of dialyzed/ lyophilized hAF.

\section{DISCUSSION}

In our study, we aimed to evaluate the feasibility and effects of lyophilization on the biological activity of hAF-borne TFs. We demonstrate that lyophilization is feasible and found partial preservation of hAF-borne TFs. Our results indicate that lyophilization preserves and even improves cellular proliferation and reduced IL-8 production. Currently, there are no studies to evaluate these effects in humans. We support the idea that hAF is a rich source of TFs that provide many benefits to the vulnerable preterm GIT.

\section{Lyophilized TF quantification}

Several studies demonstrate beneficial effect of hAF-borne TFs, especially proliferation and maturation, on the developing GIT. $^{16,19,20}$ We demonstrated that the bioactivity is highly preserved in samples subjected to dialysis/lyophilization and filtration. There are no studies examining TFs in lyophilized hAF, but a study comparing lyophilization vs cryopreservation of human amniotic membrane, another TF-rich tissue, demonstrated preservation of most TFs with some significant reduction in TFs due to lyophilization. ${ }^{21}$

Loss may be due to several factors such as fragmented proteins that may not be detected but may have some partial preservation of function. Senderoff et al. demonstrated human EGF degradation due to methionine oxidation, even at freezing temperatures and due to exposure to fluorescent lights, as well as due to succinimide formation at $\mathrm{pH}<6$ and deamidation at $\mathrm{pH}>6 .{ }^{4}$ In addition, protein may be lost due to adsorption to the membranes present in the dialysis cassettes and centrifuge filters. ${ }^{22}$ A future protein estimation assay may be helpful in determining whether protein is lost or not detected owing to conformational changes affecting antibody binding. In a potential future application of dialysis and lyophilization as a means of concentrating TFs for postnatal enteral administration, partial loss of TFs may be irrelevant as the most critical factor from a therapeutic perspective would be to deliver these factors with preserved biological activity in a minimal volume to preterm babies.

\section{Proliferation}

Several individual TFs have demonstrated trophic effects on the developing GIT, but Hirai et al. ${ }^{2}$ demonstrated that several TFs such as EGF, IGF-1, FGF, HGF, and TGF-a are comparably active. They also demonstrated that each recombinant growth factor, and even the synergistic effect of these combined growth factors, were much lower than the effects of AF alone. ${ }^{2}$ Therefore, we assert that whole hAF may be more beneficial than single TF or a simulated AF consisting of an artificial mixture of various recombinant TFs. There are likely other unknown TFs in hAF that 
may play important roles in GIT development and maturation. We have demonstrated that lyophilization of hAF preserves proliferation, despite some loss in TF quantity. Furthermore, we demonstrated that lyophilization seems to improve cellular proliferation when compared to control. In addition, hAF also contains additional vitamins, minerals, and hormones, which may work synergistically with TFs to influence GIT growth and health. ${ }^{23}$ To our knowledge, no data with hAF exist to explain the increase in bioactivity in the lyophilized hAF. In studies comparing amniotic membrane, a different yet TF-rich tissue, lyophilization showed lower TF quantity but higher graft take rate and maintained or improved healing. ${ }^{21,24,25}$ Another study demonstrating an increase in function with lyophilization as compared to cryopreservation demonstrated that cultured epidermal allografts led to greater first week healing by size in the lyophilization group. ${ }^{26}$ We also observed that lyophilization of hAF partially decreased hAF-borne TFs with increased bioavailability shown by increased IEC proliferation and reduced LPS-induced IL-8 production. We speculate that filtration performed during centrifuge microfiltration and/or dialysis prior to lyophilization removes some small compound in hAF that increases the bioavailability of the TFs or removes some inhibitors of TFs. Further in vivo and in vitro investigations are needed to determine the mechanistic pathway of increased bioavailability of hAF-borne TFs after lyophilization. In preterm infants, enteral administration of baby's own lyophilized $\mathrm{hAF}$ is feasible to simulate fetal swallowing by decreasing total volume of hAF but keeping the bioavailability of hAF. However, more studies are required before this can be achieved.

\section{Reduction of IL-8}

LPS exposure activates TLR4 in IEC via the nuclear factor-KB pathway initiating a cytokine cascade that includes increased IL-8 production. ${ }^{27} \mathrm{IL}-8$ is also a strong predictor of NEC in neonates and associated mortality ${ }^{28}$ and quantitatively can be used in predicting the severity of NEC. ${ }^{29}$ Oral administration of AF has demonstrated reduction in NEC incidence and severity via EGFmediated inhibition of TLR4 upregulation in LPS-exposed rats. ${ }^{11}$ These studies would suggest that preterm birth leading to interruption of fetal swallowing of hAF-borne TFs increases the risk for NEC in preterm neonates. Even though lyophilization of hAF led to partial decreased concentration of EGF and HGF, it significantly reduces LPS-induced IL-8 production. This again shows that lyophilization increased bioavailability of hAF-borne TFs. Lyophilization may allow for effective concentration of TFs for postnatal enteral administration as a strategy for NEC prevention in preterm infants.

\section{Limitations}

Only four TFs, namely, EGF, HGF, GM-CSF, and TGF- $a$, were analyzed and further exploration of additional TFs may be helpful. Further in vivo and in vitro studies showing increased restitution of IEC are required. Duration of lyophilized hAF-borne TF bioavailability needs to be explored. There are also no other studies evaluating lyophilization of hAF for comparison.

Future directions

The TFs in hAF may also be helpful in the recovery period from NEC. An isotonic sterile solution, a simulated AF containing recombinant human ( $r$ ) erythropoietin and rhG-CSF fed orally to neonates recovering from NEC stages IA, IB, and IIA for 3 days prior to resumption of feeds, was well tolerated as described by absence of diarrhea, abdominal distension, or guaiac-positive stools. $^{30}$ Heparin-binding EGF, another TF present in hAF, promotes restitution in post-ischemic injuries. ${ }^{31}$ Whole AF could provide these additional nutrients and potentially improve outcomes. Lyophilization and subsequent reconstitution to various concentrations of hAF-derived TFs allows feasible enteral administration to these critically ill neonates.
Preservation of $\mathrm{AF}$

Cryopreservation is a common preservation method to preserve $\mathrm{AF}$, often freezing to $-80^{\circ} \mathrm{C}$. Cryopreservation can be expensive and often unavailable, especially in developing countries. Transportation of cryopreserved tissue adds another layer of cost and complexity. Lyophilization may allow for cheaper storage and transportation at room temperature.

\section{CONCLUSION}

Dialysis/lyophilization of hAF has minimal impact on recovery of TFs and, more importantly, maintains and even improves hAF's ability to improve cellular proliferation and reduce proinflammatory responses as evidenced by reduction in IL-8 production. The mechanism is unknown and needs further investigation. Further in vivo and in vitro studies are needed to examine the effects of lyophilized hAF concentrate with partial reconstitution on TF quantity and bioactivity and to determine the smallest volumes that may be effectively reconstituted while maintaining bioactivity.

\section{AUTHOR CONTRIBUTIONS}

J.C.-manuscript author, data collection and analysis, statistical analysis. S.J.-data collection. K.S., K.R.-data collection, data analysis, manuscript revision. Y.C., K.M.data collection and analysis. S.K.J.—data analysis, manuscript investigation, principle investigator.

\section{ADDITIONAL INFORMATION}

Competing interests: The authors declare no competing interests.

Publisher's note Springer Nature remains neutral with regard to jurisdictional claims in published maps and institutional affiliations.

\section{REFERENCES}

1. $\mathrm{Ng}, \mathrm{A} . \mathrm{Y} . \mathrm{N}$. et al. Inactivation of the transcription factor Elf3 in mice results in dysmorphogenesis and altered differentiation of intestinal epithelium. Gastroenterology 122, 1455-1466 (2002).

2. Hirai, C. et al. Trophic effect of multiple growth factors in amniotic fluid or human milk on cultured human fetal small intestinal cells. J. Pediatr. Gastroenterol. Nutr. 34, 524-528 (2002)

3. Montgomery, R. K., Mulberg, A. E. \& Grand, R. J. Development of the human gastrointestinal tract: twenty years of progress. Gastroenterology 116, 702-731 (1999).

4. Senderoff, R. I. et al. Aqueous stability of human epidermal growth factor 1-48. Pharm. Res J. Am. Assoc. Pharm. Sci. 11, 1712-1720 (1994).

5. Sherbet, G. V. Growth Factors and their Receptors in Cell Differentiation, Cancer and Cancer Therapy (Elsevier, 2011).

6. Matsumoto, K. \& Nakamura, T. Hepatocyte growth factor: Molecular structure and implications for a central role in liver regeneration. J. Gastroenterol. Hepatol. 5, 509-519 (1991).

7. Walter, M. R. et al. Three-dimensional structure of recombinant human granulocyte-macrophage colony-stimulating factor. J. Mol. Biol. 224, 1075-1085 (1992).

8. Neu, J. in Probiotic Bacteria and Their Effect on Human Health and Well-Being (eds Guarino, A., Quigley, E. M. M. \& Walker, W. A.) 122-127 (Karger, Basel, 2013).

9. Leaphart, C. L. et al. A critical role for TLR4 in the pathogenesis of necrotizing enterocolitis by modulating intestinal injury and repair. J. Immunol. 179, 4808-4820 (2007).

10. Sodhi, C. P. et al. Toll-like receptor-4 inhibits enterocyte proliferation via impaired $\beta$-catenin signaling in necrotizing enterocolitis. Gastroenterology 138, 185-196 (2010).

11. Good, M. et al. Amniotic fluid inhibits Toll-like receptor 4 signaling in the fetal and neonatal intestinal epithelium. Proc. Natl Acad. Sci. 109, 11330-11335 (2012).

12. Jain, S. K. et al. Amniotic fluid-borne hepatocyte growth factor protects rat pups against experimental necrotizing enterocolitis. Am. J. Physiol. Gastrointest. Liver Physiol. 306, G361-G369 (2014).

13. Brace, R. A. Physiology of amniotic fluid volume regulation. Clin. Obstet. Gynecol. 40, 280-289 (1997). 
Lyophilization of human amniotic fluid is feasible without affecting...

$\mathrm{Jl}$ Coon et al.

852

14. Mann, S. E., Nijland, M. J. M. \& Ross, M. G. Mathematic modeling of human amniotic fluid dynamics. Am. J. Obstet. Gynecol. 175, 937-944 (1996).

15. Moore, H. M. et al. Biospecimen reporting for improved study quality (BRISQ). Cancer Cytopathol. 119, 92-101 (2011).

16. Maheshwari, A. Role of cytokines in human intestinal villous development. Clin. Perinatol. 31, 143-155 (2004).

17. Michalsky, M. P., Lara-Marquez, M., Chun, L. \& Besner, G. E. Heparin-binding EGFlike growth factor is present in human amniotic fluid and breast milk. J. Pediatr. Surg. 37, 1-6 (2001).

18. Tilton, R. G., Zhao, Y., Banerjee, S., Lejeune, W. S. \& Choudhary, S. NF-kB-inducing kinase increases renal tubule epithelial inflammation associated with diabetes. Exp. Diabetes Res. 2011, 192564 (2011).

19. Mulvihill, S. J., Stone, M. M., Fonkalsrud, E. W. \& Debas, H. T. Trophic effect of amniotic fluid on fetal gastrointestinal development. J. Surg. Res. 40, 291-296 (1986).

20. Mulvihill, S. J., Stone, M. M., Debas, H. T. \& Fonkalsrud, E. W. The role of amniotic fluid in fetal nutrition. J. Pediatr. Surg. 20, 668-672 (1985).

21. Rodríguez-Ares, M. T. et al. Effects of lyophilization on human amniotic membrane. Acta Ophthalmol. 87, 396-403 (2009).

22. Bonomini, M. et al. Proteomics characterization of protein adsorption onto hemodialysis membranes. J. Proteome Res. 5, 2666-2674 (2006).

23. Underwood, M. A. \& Sherman, M. P. Nutritional characteristics of amniotic fluid. Neoreviews 25, 341-348 (2006).
24. Ahn, J. II, Jang, I. K. \& Lee, D. H. et al. A comparison of lyophilized amniotic membrane with cryopreserved amniotic membrane for the reconstruction of rabbit corneal epithelium. Biotechnol. Bioprocess Eng. 10, 262-269 (2005).

25. Allen, C. L. et al. Augmented dried versus cryopreserved amniotic membrane as an ocular surface dressing. PLOS ONE https://doi.org/10.1371/journal. pone2013.0078441 (2013).

26. Navrátilová, Z., Slonková, V., Semrádová, V. \& Adler, J. Cryopreserved and lyophilized cultured epidermal allografts in the treatment of leg ulcers: a pilot study. J. Eur. Acad. Dermatol. Venereol. 18, 173-179 (2004).

27. Nanthakumar, N. N., Fusunyan, R. D., Sanderson, I. \& Walker, Wa Inflammation in the developing human intestine: A possible pathophysiologic contribution to necrotizing enterocolitis. Proc. Natl Acad. Sci. USA 97, 6043-6048 (2000).

28. Benkoe, T. et al. Interleukin-8 predicts 60 -day mortality in premature infants with necrotizing enterocolitis. J. Pediatr. Surg. 49, 385-389 (2014).

29. Benkoe, T. et al. Interleukin 8 correlates with intestinal involvement in surgically treated infants with necrotizing enterocolitis. J. Pediatr. Surg. 47, 1548-1554 (2012).

30. Lima-Rogel, V. et al. Tolerance of a sterile isotonic electrolyte solution containing select recombinant growth factors in neonates recovering from necrotizing enterocolitis. J. Perinatol. 23, 200-204 (2003).

31. Feng, J. \& Besner, G. E. Heparin-binding epidermal growth factor-like growth factor promotes enterocyte migration and proliferation in neonatal rats with necrotizing enterocolitis. J. Pediatr. Surg. 42, 241-20. (2007). 